\title{
Neural networks technique for detecting current systems while main phase of geomagnetic storm
}

\author{
N. A. Barkhatov, S. E. Revunov, O. T. Cherney, M. V. Mukhina, and Zh. V. Smirnova
}

Minin Nizhny Novgorod State Pedagogical University (Minin University), Nizhny Novgorod, Russian

\begin{abstract}
The study demonstrated the technology for searching nonlinear correlations between the intensity indices of auroral electric jets (AU, AL) and ring current intensity indices (SYM, ASY). The wellknown connection of the western electrojet with the asymmetric part of the ring current and the eastern electrodett with the symmetric is confirmed. The technology of artificial neural networks was chosen as the main method. Thus, the relationship of magnetic disturbances in the auroral region and magnetic disturbances at middle and low latitudes in the main phase of a geomagnetic storm is investigated. The characteristic lead times in the development of mid-latitude magnetospheric processes relative to polar are determined. An acceptable neural network recovery of auroral electrojet intensity indices from ring current index data is demonstrated.
\end{abstract}

\section{Introduction}

Within the framework of classical representations about development of ionospheric and magnetospheric current systems there is a set of models which describe their evolution depending on a level of geomagnetic activity. However the problem of development and unification of these current systems by present time remains still not solved. In this connection research of space-time behaviour of a geomagnetic field and construction of the corresponding theoretical models describing process of generation, development and collapse of current systems in terrestrial magnetosphere proceeds.

In [1-6] the search of linear connection between indices describing development of symmetric (SYM) and asymmetric (ASY) parts of a ring current with indices of eastward (AU) and westward (AL) electrojets intensity has been made. On the basis of the correlation connections analysis between the specified indices on the main phase of a geomagnetic storm it has been established, that the symmetric part of a ring current develops synchronously with eastward electrojet and is behind in development from westward electrojet about 2 hours. The asymmetric part of a ring current has connection with both electrojets and during the main phase of a geomagnetic storm develops with them synchronously.

The present research is sequential of the work [7-10]. At that work the connection between indices of ring current and auroral electrojets intensity was studied by the example of concrete geomagnetic storms. Basic difference of the given research that the general tendencies of indices relationship between storms of various intensity are analyzed. Besides as the tool for search of connection of ring current elements with auroral electrojets it is used Elman's artificial neural network [11-14] with which help its nonlinear character comes to light (fig. 1). The developed method helps to reply the question - whether there are general laws in development of geomagnetic storms, and as consequence, whether is possible to recovery of auroral electrojets intensity indices according to indices of a ring current intensity on the main phase of a geomagnetic storm.

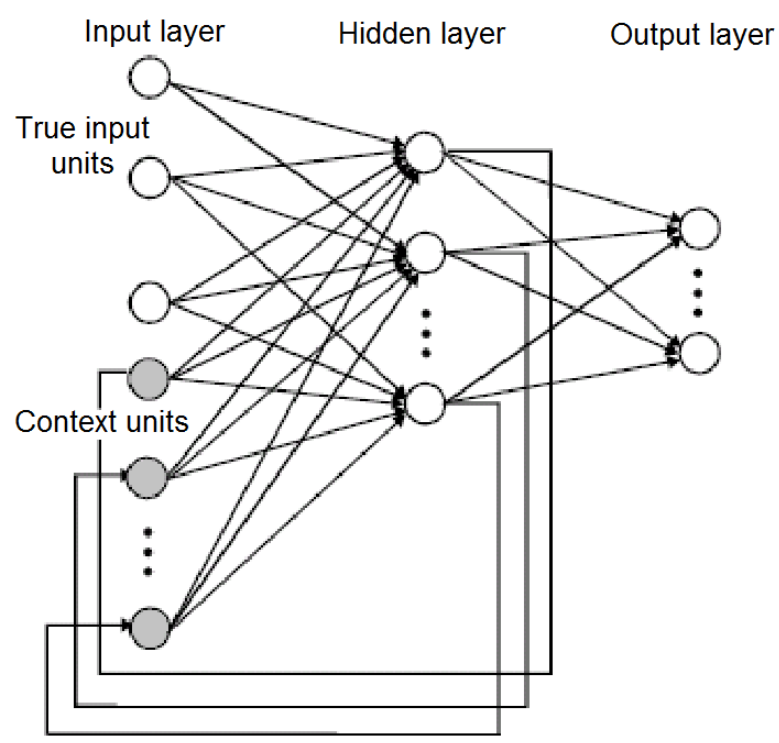

Fig. 1. Elman ANN architecture.

\section{Data and research technique}

It have been selected 15 geomagnetic storms of various intensity $(-300 \leq$ Dst $\leq-50 \mathrm{nT})$ for the period with 2000 
on 2001 for investigation of nonlinear connections between pairs of indices SYM-AU, SYM-AL, ASY-AU and ASY-AL. Selection of events was carried out on Dst index. In sample those geomagnetic storms got only, which form corresponded to their classical development (Fig. 2).

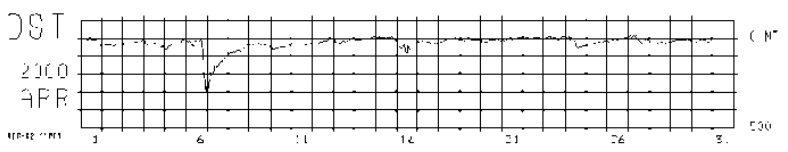

Fig. 2. A time behavior of Dst index in April, 2000. It has been selected April, 6 and 24 storms for the present research.

Each of the chosen events has been divided on the main phase and a recovery phase of geomagnetic storm on values of Dst index. In offered research nonlinear connection between current systems was established only on the main phase of geomagnetic storm cause during this period a ring current and auroral electrojets are most developed.

The search of such connection between the noted pairs of indices was carried out according to sequence:

For elimination of «neural network jitter» general
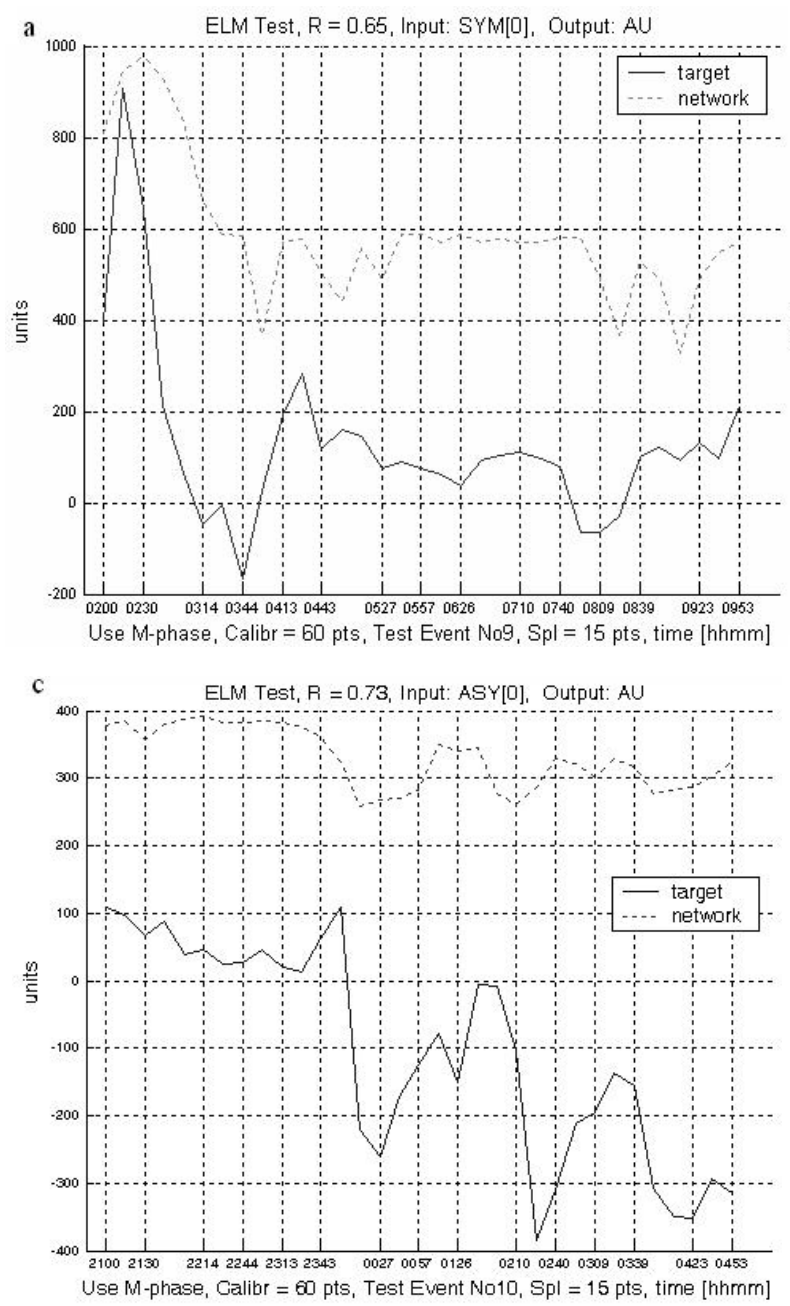

time of averaging for all examined indices got out. In this case it made 15 minutes. Such «ANN jitter» frequently arises because of the large amplitude sharp peaks in AU and AL indices. Classical fifteen-minutes averaging allows to smooth such peaks and to reveal the general tendency in development of auroral current systems.

At the given stage the training of neural network on one of considered events was carried out. During training there was a weights adjustment, i.e. the neural network established connection between indices SYM, ASY and indices AU, AL within the limits of a concrete geomagnetic storm.

With the help of the neural network already trained on this event, connections between pairs of indices SYM-AU, SYM-AL, ASY-AU and ASY-AL for the others 14 events were examined. For this purpose data recovery of indices AU, AL on values of indices SYM, ASY was carried out. Data recovery was accepted effective at reception of linear correlation between real and recovered sequence of the greater 0.5 . Presence of high linear correlation in this case showed, that connection between mid-latitude and polar current systems has the same character, as in event on which
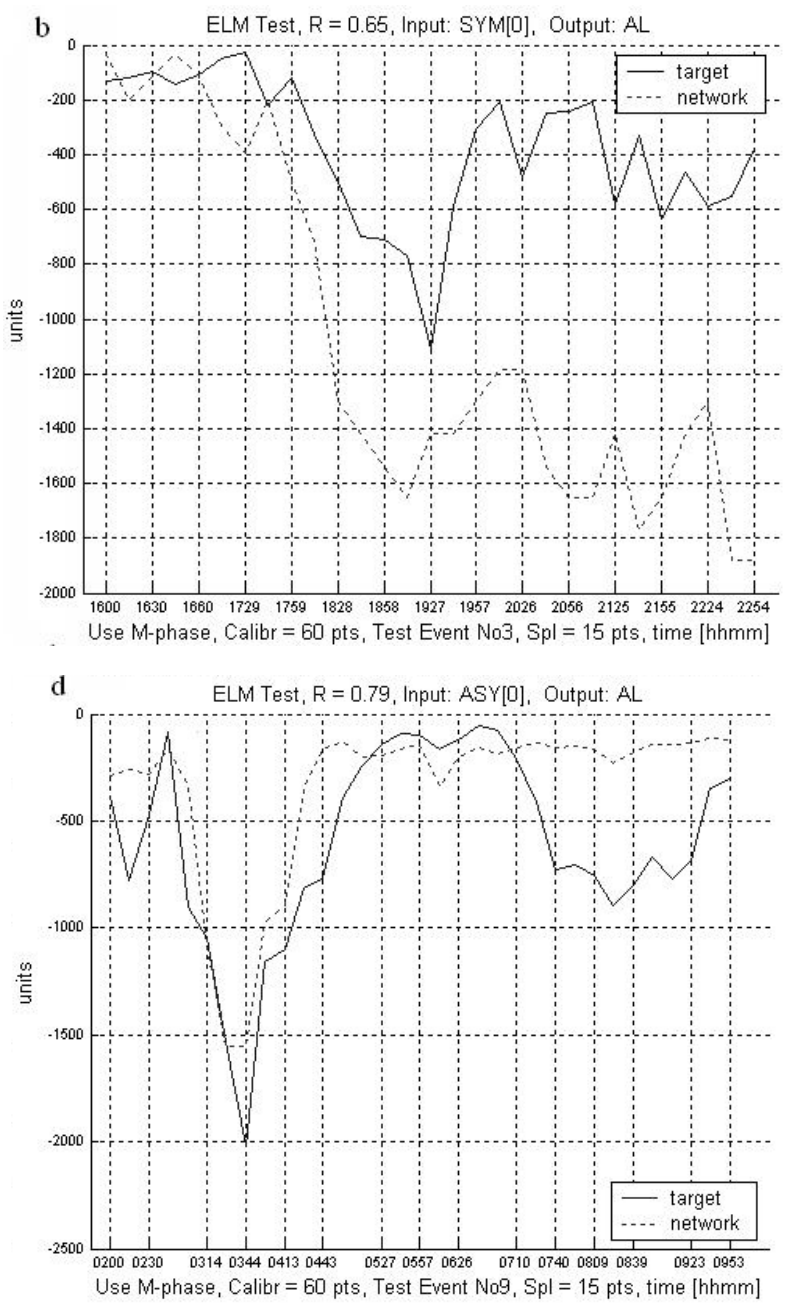

Fig. 3. Examples of data recovery of index AU on index SYM (a); index AL on index SYM (b); index AU on index ASY (c); index AL on index ASY (d). 
there was training. Absence of linear correlation speaks that in new event character of connection is essentially other. Thus it is possible to determine a distinction in development united ionosphere-magnetospheric current system for different geomagnetic storms.

According to this scheme all 15 events are considered. It is necessary to note, that at neural network experiments for improvement of data recovery quality the delay time of development mid-latitude current systems relatively auroral current systems was entered. For SYM-AU pair it has on the average made 0.5 hours, and for SYM-AL pair - 2 hours [1]. For other pairs of indices time of such delay appeared equal to zero.

\section{Results of numerical experiments}

As a result of neural network experiments it is revealed, that acceptable data recovery of eastward and westward electojets intensity indices according to indices of a symmetric and asymmetric parts of ring current is possible. Entering of a delay in development midlatitude current systems relatively polar current systems in some cases essentially improves data recovery quality for AU and AL indices on SYM index data. It means in the general case that for a ring current it is typical to have a delay in development relatively auroral electrojets for geomagnetic storms of different intensity.

Examples of auroral electrojets intensity indices data recovery on ring current intensity indices for each of considered pairs are given on Fig. 3.

The establishment of characteristic dependences in development of examined current systems is possible by carrying out of the statistical analysis of neural network data recovery results. For this purpose the weight of number of satisfactory correlation cases for each pair of indices (SYM-AU, SYM-AL, ASY-AU, ASY-AL) in the general number of possible events combinations is calculated:

$$
W=\frac{N}{C_{n}^{m}},
$$

where $N$ - number of cases with correlation coefficient $>$ $0.5, C_{m}^{n}-$ the general number of events combinations from $m$ on $n$. Values of weights $W$ for each pair of indices is shown on Fig. 4.

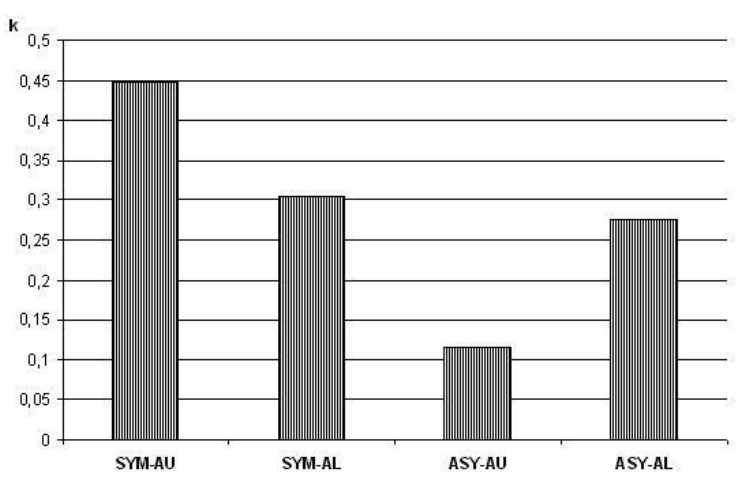

Fig. 4. Values of weight $W$ of number of satisfactory correlations for each pair of considered indices.

\section{Conclusion}

Thus, the statistical analysis of recovery data results shows, that for SYM-AU and ASY-AL index pairs the greatest quantity of events with correlation coefficient > 0.5 between real and recovered sequences is observed. It means, that current systems of a symmetric part of a ring current and eastward electrojet as well as current systems of an asymmetric part of a ring current and westward electrojet have steady connection and the similar scenario of development within the limits of geomagnetic storms of different intensity.

In the first case (for SYM-AU pairs) it can be caused by activation considered current systems by a unified source. Indeed current system SYM is self-contained system and does not require association with eastward electrojet AU. For ASY-AL pairs appreciable correlation can speak about association of their current systems. Such conclusion makes agree to results of works [1-6]. The received low correlation level for ASY-AU pairs also does not contradict researches [7-9, 12, 15]. Suggested research is a basis for an establishment of the general interrelation of ionosphere-magnetospheric processes at different phases of storm development with changes in parameters of the interplanetary space.

\section{References}

1. L.D. Landau, E.M. Lifshits. Theoretical Physics: Electrodynamics of Continuous Media, 8, (Moscow, Ed.Nauka, 624, 1982) (in Russian)

2. K.I. Gringauz, I.A. Zhulin, Physics of the magnetosphere (Moscow, Ed. Mir, 592, 1972) (in Russian)

3. Yu.V. Manakova, K.A. Pekhteleva, N.A. Barkhatov, S.E. Revunov, Space-time analysis of perturbations of the Pc4-5 range during periods of magnetic storms by the correlationskeleton method, Vestnikof Minin University 1, 1-6 (2016)

4. N.A. Barkhatov, Development of methods for predicting the geomagnetic state of the magnetosphere based on the search for fundamental laws of solar-terrestrial 
relationships, Vestnikof Minin University 2, 111 (2013)

5. N.A. Barkhatov, S.E. Revunov, A.B. Vinogradov, The classification algorithm for MHD wavelet-skeleton spectral patterns of geoeffective plasma flows in the solar wind, Vestnikof Minin University 3, 1-6 (2014)

6. N.A. Barkhatov, V.G. Vorobjev, S.E. Revunov, O.M. Barkhatova, E.A. Revunova, O.I. Yagodkina, Neural network classification of substorm geomagnetic activity caused by solar wind magnetic clouds, Journal of Atmospheric and Solar-Terrestrial Physics 205 (2020) 105301, DO1 10.1016/j.jastp.2020.105301

7. N.A. Barkhatov, S.E. Revunov, Zh.V. Smirnova, O.T. Cherney, E.A. Semahin, Neural Network Classification of Space Plasma Parameter Discontinuities, International Journal of Advanced Trends in Computer Science and Engineering 9, 3 (May-June 2020)

8. N.A. Barkhatov, S.E. Revunov, O.M. Barkhatova, E.A. Revunova, N.V. Kosolapova, Algorithm for Detecting an Extreme Solar Flare of September 6, 2017 from its Geomagnetic Precursors, Radiophysics and Quantum Electronics 62, 10, 651-656 (March, 2020) (Russian Original 62, 10, October 2019), DOl 10.1007/s11141-020-10010-6

9. N.A. Barkhatov, S.E. Revunov, V.G. Vorobjev, O.I. Yagodkina, Studying the relationship between high-latitude geomagnetic activity and parameters of interplanetary magnetic clouds with the use of artificial neural networks, Geomagn. Aeron. 58, 2, 147-153 (2018), DOl $10.7868 /$ S0016794018020013

10. N.A. Barkhatov, S.E. Revunov, Z.V. Smirnova, O.T. Cherney, Observing of polar cap dynamic by Greenland chain of magnetic stations, International Journal of Emerging Trends in Engineering Research 8, 5, 1552-1555 (May 2020), DOI 10.30534/ijeter/2020/13852020

11. W.K.M. Rice, J.P. Zank, G. Lee, Particle acceleration and coronal mass ejection driven shocks: Shocks of arbitrary strength, J. Geophys. Res. 108, A10, 1369 (2003), DOI 10.1029/2002JA009756

12. B.U.O. Sonnerup, S. Haaland, G. Paschmann, B. Lavraud, M.W. Dunlop, H. Reme, A. Balogh, Orientation and motion of a discontinuity from single-spacecraft measurements of plasma velocity and density: Minimum mass flux residue, J. Geophys. Res. 109, A03221 (2004), DOI 10.1029/2003JA010230

13. S. Haaland, B.U.O. Sonnerup, M.W. Dunlop, E. Georgescu, G. Paschmann, B. Klecker, A. Vaivads, Orientation and motion of a discontinuity from Cluster curlometer capability: Minimum variance of current density, Geophys. Res. Lett. 31, L10804 (2004), DOI: $10.1029 / 2004 G L 020001$

14. B.U.O. Sonnerup, H. Hasegawa, Orientation and motion of two-dimensional structures in a space plasma, J. Geophys. Res. 110, A06208 (2005), DOI: 10.1029/2004JA010853

15. J. Merka, A. Szabo, J.A. Slavin, M. Peredo, Three-dimensional position and shape of the bow shock and their variation with upstream Mach numbers and interplanetary magnetic field orientation, J. Geophys. Res. 110, A04202 (2005), DOI: 10.1029/2004JA010944. 